\title{
Scheimpflug vs Scanning-Slit Corneal Tomography: Comparison of Corneal and Anterior Chamber Tomography Indices for Repeatability and Agreement in Healthy Eyes
}

This article was published in the following Dove Press journal: Clinical Ophthalmology

\begin{abstract}
Anastasios John Kanellopoulos ${ }^{1,2}$
'Department of Ophthalmology, The LaserVision Clinical and Research Eye Institute, Athens, Attiki, Greece; ${ }^{2}$ Department of Ophthalmology, New York University Medical School, New York City, NY, USA
\end{abstract}

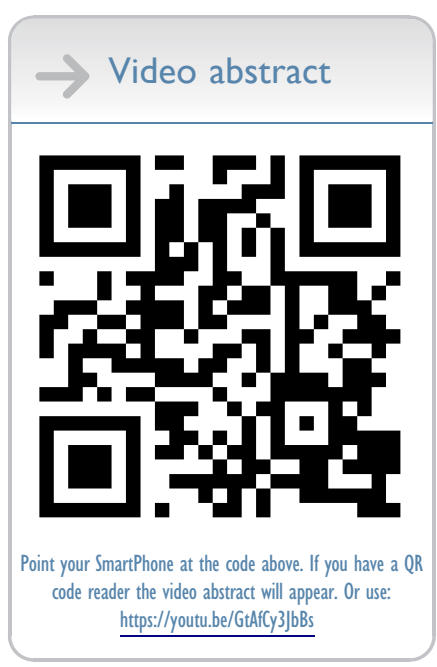

Correspondence: Anastasios John Kanellopoulos

The LaserVision Clinical and Research Eye Institute, Athens, Greece

Tel + 302107472777

$\mathrm{Fax}+302107472789$

Email ajkmd@mac.com
Purpose: To evaluate and compare the repeatability and agreement of Scheimpflug vs scanning-slit tomography of the cornea and the anterior chamber in terms of keratometric and tomographic indices in healthy eyes.

Methods: The 20 eyes of 10 healthy participants underwent 3 consecutive measurements using both Scheimpflug-tomography and scanning-slit tomography, diagnostic devices. Multiple corneal and anterior chamber tomographic parameters were recorded and evaluated to include corneal keratometry and its axis; corneal best-fit sphere (BFS), pachymetry mapping, angle kappa, anterior chamber depth (ACD), pupil diameter, and location. Repeatability for each device was assessed using the within each subject standard deviation of sequential exams, the coefficient variation (CV) and the intraclass correlation coefficient (ICC). Agreement between the two devices was assessed using Bland-Altman plots with 95\% limits of agreement (LoA) and correlation coefficient (r). Results: Both devices were found to have high repeatability $(\mathrm{ICC}>0.9)$ both in keratometric and other tomographic measurements. Scheimpflug tomography's repeatability though appeared superior in the average keratometry values, anterior and posterior BFS, thinnest corneal pachymetry value and location $(\mathrm{p}<0.05)$. Agreement: Statistically significant interdevice differences were noted in the mean values of K1, K2, BFS, ACD and thinnest corneal pachymetry $(\mathrm{p}<0.05)$. Despite the agreement differences noted, the two devices were well correlated $(r>0.8)$ in respective measurements with Scheimpflug delivering consistently lower values than the scanning-slit tomography device.

Conclusion: Scheimpflug-tomography repeatability was found to be superior to that of scanning-slit tomography in this specific study, in most parameters evaluated. Inter-device agreement evaluation suggests that reading from the two devices may not be used interchangeably in absolute values, yet they are well correlated with Scheimpflug delivering consistently lower values in most.

Keywords: Pentacam, Orbscan, Scheimpflug corneal tomography, scanning-slit corneal tomography

\section{Introduction}

As new devices for measuring anterior segment parameters become available in the clinical practice, the need to compare measurements obtained using various different technologies for healthy and pathologic eyes emerges. By comparing intra-device agreement, it can be determined whether different 
devices can be used interchangeably or whether their readings are consistently correlated. The inter-device repeatability must also be assessed to determine how much of a deviation from normal, or average, represents true pathology and the magnitude of change required to be considered progression of disease rather than variability due to test re-test variance.

Corneal tomography has been evolving in the recent years with Scheimpflug-based tomography being the possible successor to scanning-slit tomography. Comparison in a healthy group of subjects should aim to evaluate their relative intra-device repeatability as well as their inter-device agreement. Having established comparisons in healthy corneas first, results could then function as a benchmark for studying pathologic corneas.

The clinical implications of the accuracy of these values is paramount, first considering that they are used clinically to define healthy from diseased, a task that we have previously reported on extensively. ${ }^{1-15}$ Furthermore, refractive surgery both corneal and lens-based, and especially therapeutic-based refractive laser interventions, have long involved corneal, anterior chamber and pupillary imaging by evolving tomographic devices, as a means of customization of surgical accuracy and its effective assessment. We have also reported extensively on corneal imaging and specifically tomographic imaging used in naïve and/or irregular corneas as a customized-therapeutic surgical intervention. ${ }^{16-37}$

To date, comparisons between Scheimpflug and scanning-slit tomography devices in healthy corneas mainly focused on pachymetric measurements, showing variable results in terms of device agreement: some with good agreement, some with moderate agreement and others with poor agreement having been reported. ${ }^{38-42}$

Fewer studies have compared Scheimpflug and scanning-slit devices in keratometric measurements on healthy corneas, though all have found poor agreement. ${ }^{40,43-45}$

We herein present a prospective study of 20 eyes of 10 healthy subjects, comparing the intra-device repeatability and assessing the inter-device agreement of Scheimpflug vs Scanning-Slit tomography in terms of keratometric and topometric readings for the cornea and the anterior chamber.

\section{Methods}

This prospective comparative study was approved by the Ethics Committee of our Institution (Laservision Clinical and Research Eye Institute) and adhered to the tenets of the Declaration of Helsinki. This study was conducted in the clinical practice of Laservision, Clinical and Research Eye Institute. Inclusion criteria were healthy eyes with no other ocular pathology other than refractive error. Written informed consent was provided by all participants of the present study.

A Scheimpflug-tomography imaging device (model: Pentacam HRsystem, Oculus, Wetzlar, Germany) and a scanning-slit imaging device (model: Orbscan II, Bausch \& Lomb, Rochester, NY) were employed. For each eye, three consecutive measurements were taken with each device. Subjects were instructed to blink immediately prior to each measurement. No eyedrops were applied prior to testing.

\section{Scheimpflug-Tomography Imaging}

More recently available corneal tomographers rely on the Scheimpflug-principle whereby imaging with a wide depth of focus allows a planar object that is not parallel to the image plane to be in focus. The specific Scheimplug-tomography device used in our study was the Pentacam HRsystem (Oculus, Wetzlar, Germany), that uses a single, rotating Scheimpflug camera, and monochromatic slit-light source, in combination with a static camera, in order to obtain 500 measurement points, from a total of 50 single slitimages, corresponding to specific angles along the optical axis, totaling 25,000 measurement points, and thus to generate a 3-dimensional image of the cornea. A quality score is provided for each scan, with an "OK" given for acceptable scans. ${ }^{46}$

\section{Scanning-Slit Imaging}

The Orbscan II device (Bausch \& Lomb, Rochester, NY) was the scanning-slit tomography device used in our study, and uses both scanning-slit and Placido-disc technology to combine keratometry measurements with assessment of the anterior and posterior corneal surfaces which allows a three-dimensional reconstruction of the cornea. ${ }^{47}$ The device projects 40 slits, 20 from each side, onto the cornea and records the backscattered light. The Orbscan II software does not provide 
a quality score; instead, it automatically assesses and discards measurements deemed to be of unacceptable quality.

\section{Corneal and Anterior Segment Parameters Studied}

Parameters studied with both devices included corneal keratometry: flat keratometry (K1), steep keratometry (K2), and maximum keratometry (Kmax), as well as the relevant astigmatic power for the central $5 \mathrm{~mm}$ of the corneas studied, and its relevant axis; anterior and posterior corneal best-fit sphere (BFS), thinnest corneal point and its topographic location in regard to the corneal vertex, as well as the corneal vertex topographic location in regard to the center of the pupillary aperture and thus the hypothetical eye-geometric center, illustrated in the imaging background ( $\mathrm{x}, \mathrm{y}$ coordinates of angle kappa). Additionally, other anterior chamber tomographic values such as anterior chamber depth (ACD), pupil diameter, as well as its topographic relation of the pupillary center in regard to the superimposed cornea vertex and hypothetical line-of-sight.

\section{Statistical Analysis}

Repeatability, defined as test-retest variability, was evaluated using the within-subject standard deviation (SD), within-subject coefficient variation (CV) and the intraclass correlation coefficient (ICC). The withinsubject $\mathrm{CV}$ was calculated as the SD divided by the overall mean and expressed as a percentage. ICC is defined is the ratio of the between-subject variance to the sum of the within and between-subject variance, ranging from 0 to 1 , with 1 indicating perfect repeatability. Paired $t$-test was used to compare the repeatability of the scanning-slit device to that of the Scheimpflug-based device. Statistical $\mathrm{p}$ values $<0.05$ were considered significant.

Agreement between devices (inter-device agreement) was assessed using Bland-Altman plots, ${ }^{11,12}$ the correlation coefficient (r) and a two tail $t$-test. Bland-Altman plots were generated by plotting the difference of the measurements from each device against their mean. Limits of agreement (LoA) were set at $95 \%$ (mean difference $\pm 1.96 \mathrm{SD}$ ), defining the range within which most differences between measurements from the two devices will lay.

\section{Results}

Twenty eyes of 10 healthy subjects were included in this prospective comparative study. The mean age was $40.9 \pm$ 22.7 years ( 8 females, 2 males). No eyes were excluded based on the quality of the scans. Figure 1 illustrates the images evaluated with both technologies in a random eye of the patients studied: all 3 scanning-slit tomography individual scans as well as all 3 respective Scheimpflug scans for that specific eye are included.

\section{Repeatability}

In terms of keratometric measurements, both devices were found to have high repeatability (ICC $>0.9$ ) in all keratometric readings except for the $\mathrm{x}$ and $\mathrm{y}$ axis of the kappa intercept $(\mathrm{ICC}<0.9)$. Scheimpflug tomography was found to be superior in repeatability to scanning-slit tomography in mean $\mathrm{K} 2(\mathrm{P}=0.034)$ and mean $\mathrm{K} 1(\mathrm{P}=0.027)$ while the two devices' repeatability showed no statistically significant difference in maximum kappa angle, $\mathrm{x}$ and $\mathrm{y}$ axis of the kappa intercept and the mean corneal power at the $5.0 \mathrm{~mm}$ zone.

The evaluation of the best-fit sphere, both devices showed high repeatability $(\mathrm{ICC}>0.9)$ with a superiority for Scheimpflug in both anterior $(\mathrm{P}=0.021)$ and posterior $(\mathrm{P}=0.039)$ BFS. Both devices were also found to have high repeatability in ACD, astigmatic power at the $5.0 \mathrm{~mm}$ zone, steep axis and pupil diameter (ICC $>0.9$ ) with no statistically significant difference between them $(\mathrm{P}>0.05)$. Of note, steep axis had a remarkably lower ICC in both devices Scheimpflug being superior to slit scan (ICC: 0.7 vs 0.55 ).

Similarly, in terms of pachymetric measurements, both devices were found to have high repeatability measuring the thinnest cornea thickness (ICC $>0.9$ ) with Scheimpflug appearing superior to the scanning slit $(\mathrm{P}=0.038)$. When comparing thinnest location, Scheimflung's repeatability was statistically significantly higher both in the $\mathrm{x}$ axis $(\mathrm{P}=0.043)$ and $y$ axis $(\mathrm{P}=0.031)$ with ICC of 0.92 for the $\mathrm{x}$ axis and 0.87 for the $\mathrm{y}$ axis vs 0.43 and 0.65 for the scanning slit, respectively. These data are summarized in Table 1.

\section{Agreement}

Agreement was assessed using Bland-Altman plots for keratometric indices (Figure 2), pachymetric indices (Figure 3) and the rest of the parameters studied (Figure 4). 
All keratometric measurements were found to be well correlated between the two devices $(r>0.8)$ with the exception of the kappa intercept $\mathrm{x}(\mathrm{r}=0.26)$ and $y$ axis $(r=0.17)$. Steep and flat keratometry yielded a statistically significant difference between the two devices $(\mathrm{P}=0.022$ and $\mathrm{P}=0.04$, respectively), with Scheimpflug readings being consistently lower than those derived from the scanning-slit device.

The anterior BFS was found to be different among devices $(\mathrm{P}=0.032)$ while it showed high correlation $(\mathrm{r}=0.96)$. Posterior BFS had no significant difference $(\mathrm{P}>0.05)$ and $\mathrm{r}=0.93$.
Different values were procured between the devices for anterior chamber depth (ACD), astigmatic power and pupil diameter $(\mathrm{P}<0.001)$. ACD had the highest $\mathrm{r}$ among these parameters $(r=0.99)$ while the rest proved lower than 0.9 . Specifically, $r=0.21$ for astigmatic power, $r=0.83$ for pupil diameter and $\mathrm{r}=0.51$ for steep axis.

Thinnest corneal thickness was found to be different between the two devices $(\mathrm{p}<0.001)$ with an $\mathrm{r}=0.99$. The $\mathrm{x}$ axis value was also found to be statistically significant different between the devices $(\mathrm{P}=0.003)$ and $\mathrm{r}=0.66$. The $\mathrm{y}$ axis value was not different and had an $\mathrm{r}=0.23$. All keratometric and pachymetric readings evaluated are
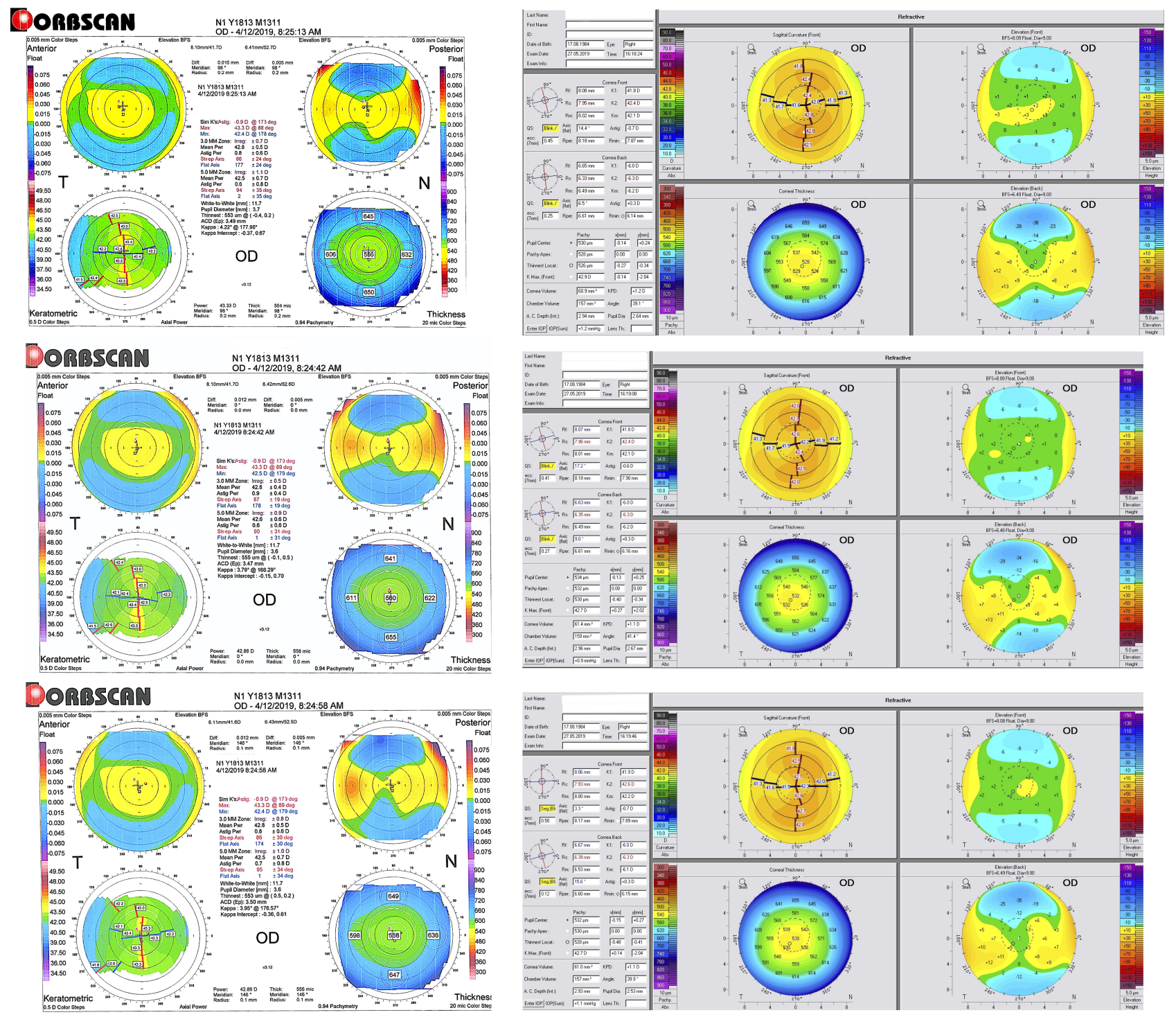

Figure I This image illustrates the scans evaluated with both technologies in a random eye of one the patients studied: all 3 scanning-slit tomography individual scans (on the left) as well as all 3 respective Scheimpflug scans (on the right) for that specific eye are included. 
Table I Summary of the Comparative Statistical Analysis of the Parameters Studied

\begin{tabular}{|c|c|c|c|c|c|c|c|}
\hline & $\begin{array}{l}\text { SD } \\
\text { Pentacam }\end{array}$ & $\begin{array}{l}\text { SD } \\
\text { Orbscan }\end{array}$ & $\begin{array}{l}\text { CV (\%) } \\
\text { Pentacam }\end{array}$ & $\begin{array}{l}\text { CV (\%) } \\
\text { Orbscan }\end{array}$ & $\begin{array}{l}\text { ICC } \\
\text { Pentacam }\end{array}$ & $\begin{array}{l}\text { ICC } \\
\text { Orbscan }\end{array}$ & $\mathbf{P}$ \\
\hline K2 steep keratometry & 0.11 & 0.17 & 0.25 & 0.39 & 0.99 & 0.98 & 0.034 \\
\hline $\mathrm{KI}$ flat keratometry & 0.10 & 0.16 & 0.23 & 0.37 & 0.99 & 0.98 & 0.027 \\
\hline Kmax Steepest & 0.13 & 0.23 & 0.30 & 0.54 & 0.98 & 0.96 & $>0.05$ \\
\hline $\mathrm{X}$ angle kappa & 0.23 & 0.13 & 193.78 & 31.91 & 0.52 & 0.72 & $>0.05$ \\
\hline Y Angle kappa & 1.29 & 0.12 & 170.24 & 209.14 & 0.40 & 0.85 & $>0.05$ \\
\hline $\mathrm{K}$ mean power & 0.10 & 0.14 & 0.23 & 0.34 & 0.98 & 0.98 & $>0.05$ \\
\hline BFS front & 0.01 & 0.02 & 0.08 & 0.25 & 0.99 & 0.98 & 0.021 \\
\hline BFS posterior & 0.02 & 0.03 & 0.30 & 0.45 & 0.99 & 0.98 & 0.039 \\
\hline$A C D$ & 0.01 & 0.01 & 0.43 & 0.29 & 0.99 & 0.99 & $>0.05$ \\
\hline Astig power & 0.08 & 0.12 & 14.33 & 16.85 & 0.98 & 0.93 & $>0.05$ \\
\hline Steep Axis & 6.89 & 6.11 & 9.47 & 6.86 & 0.7 & 0.55 & $>0.05$ \\
\hline Pupil Diameter & 0.09 & 0.10 & 0.57 & 0.97 & 0.8 & 0.98 & $>0.05$ \\
\hline Thinnest pachymetry & 3.19 & 5.73 & 0.57 & 0.97 & 0.99 & 0.99 & 0.038 \\
\hline $\begin{array}{l}\text { Thinnest } x \text { from } \\
\text { vertex }\end{array}$ & 0.07 & 0.23 & 17.31 & 91.08 & 0.92 & 0.43 & 0.043 \\
\hline $\begin{array}{l}\text { Thinnest y from } \\
\text { vertex }\end{array}$ & 0.07 & 0.23 & 47.59 & 159.59 & 0.87 & 0.65 & 0.031 \\
\hline
\end{tabular}

shown in Tables 1 and 2 respectively along with their repeatability and agreement indices for each device.

\section{Discussion}

This comprehensive prospective comparative analysis of multiple keratometric and tomography measurements employing various statistical analysis indices such as within subject standard deviation (SD), within subject coefficient variation $(\mathrm{CV})$ and intraclass correlation coefficient (ICC) for repeatability and BlandAltman plots, correlation coefficient (r) and a two-tail $t$-test for agreement.

Our results suggest high repeatability of both Scheimpflug and Scanning-slit devices as previously reported in the literature. ${ }^{50}$ In the current study, Scheimpflug tomography's repeatability was found to be superior in mean $\mathrm{K} 1$, and $\mathrm{K} 2$ keratometry, anterior and posterior best-fit sphere, and thinnest corneal thickness and location. $(p<0.05)$. The higher test-retest variability of the scanning-slit measurements suggests that a significantly higher threshold for change, may be required to be considered genuine progression compared to the Scheimpflug device studied (Pentacam HR). ${ }^{51}$

Inter-device poor agreement in our study suggests the two devices may not be used interchangeably in absolute values measured; yet they were found to be well correlated with Scheimpflug delivering consistently lower values in K1, K2 keratometry, best-fit sphere, anterior chamber depth, and thinnest corneal thickness. These findings are in accordance with previous reports suggesting poor agreement between the devices in keratometric indices. ${ }^{52}$ Our results on pachymetric measurements are in agreement with those reported by Sedaghat et al, ${ }^{53}$ while other studies have reported thinner pachymetric readings with the 

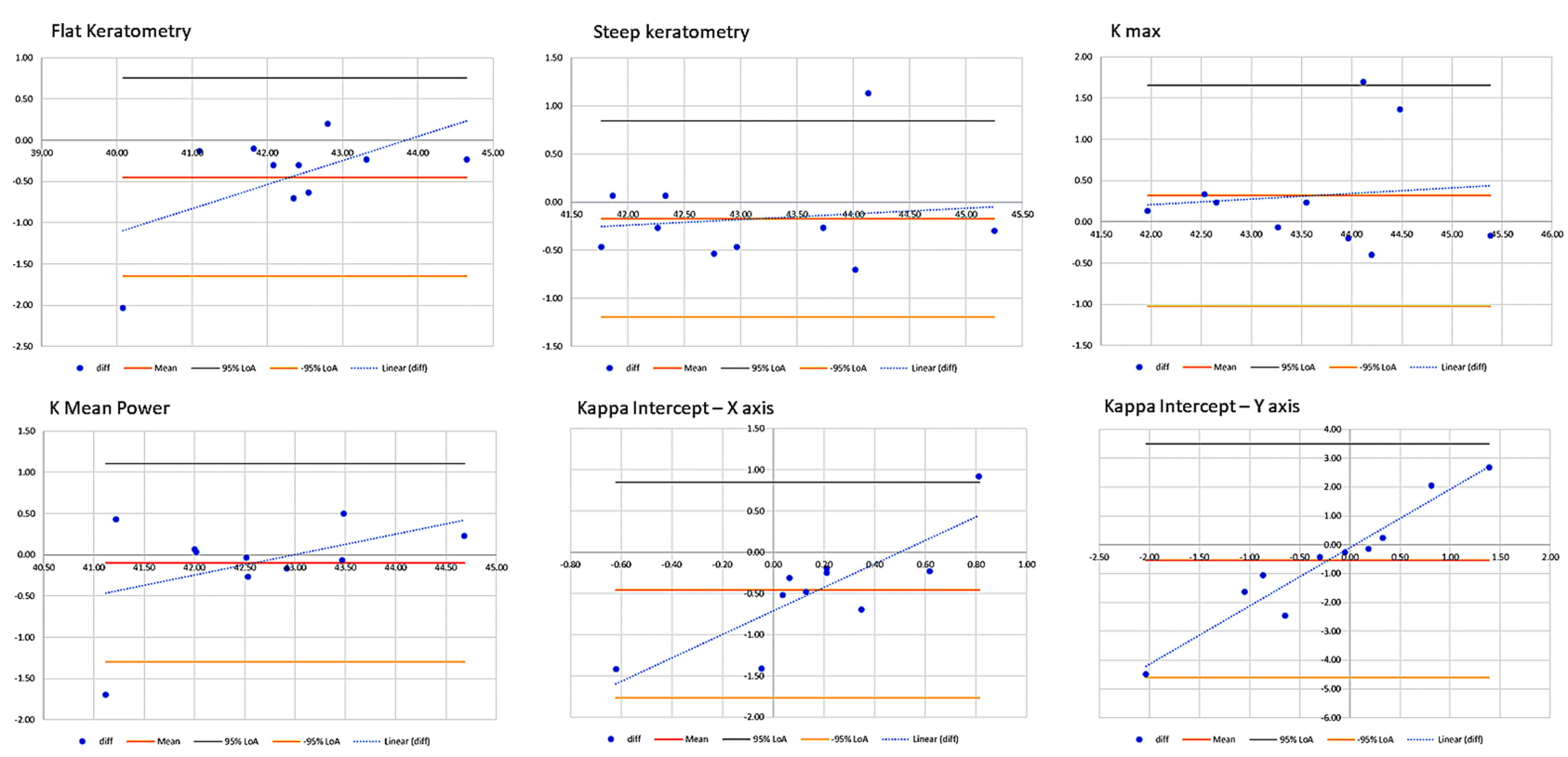

Figure 2 This image illustrates the Bland-Altman plots for the keratometric indices analyzed statistically.
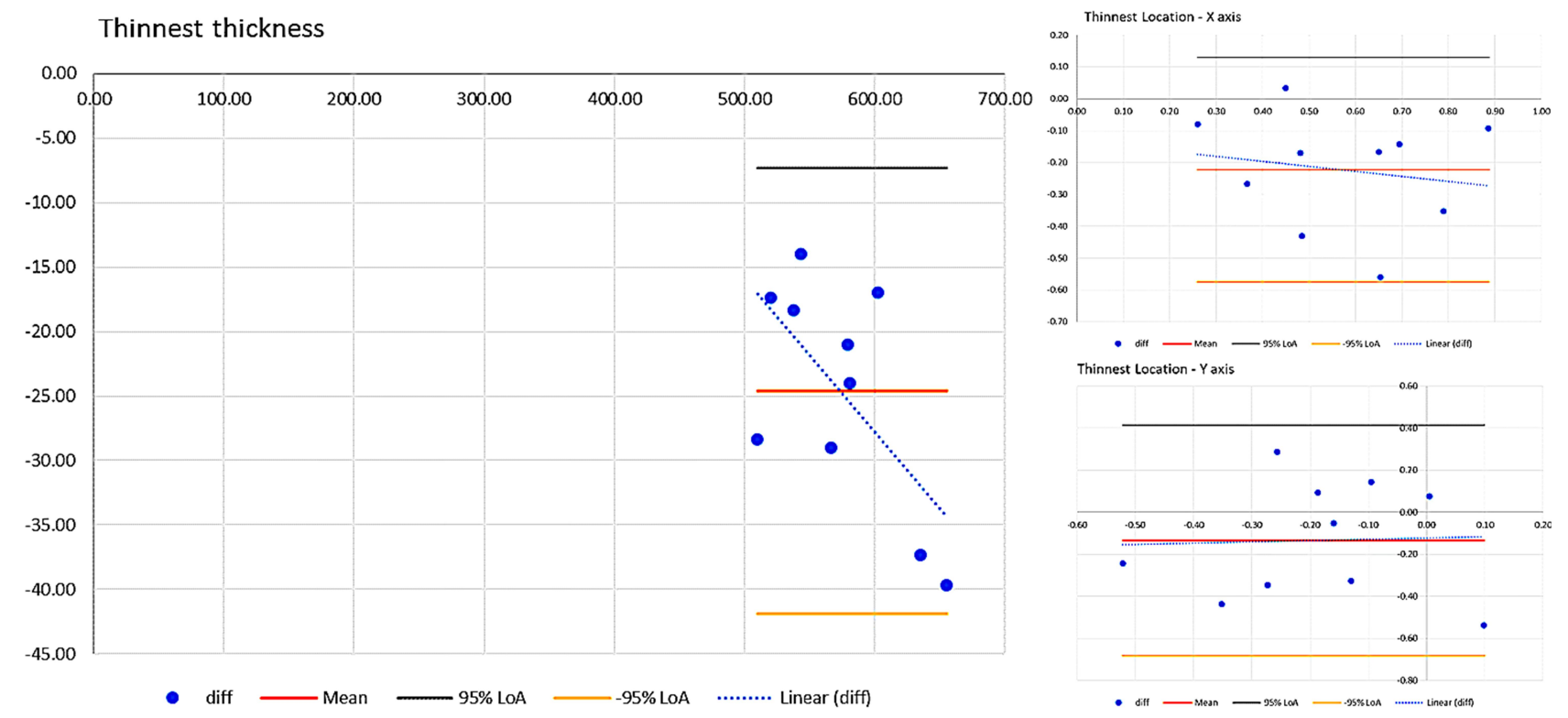

Figure 3 This image illustrates the Bland-Altman plots for the pachymetric indices analyzed statistically.

Orbscan $\mathrm{II}^{39,54,55}$ or no statistically significant differences. ${ }^{56,57}$

These findings have clinically relevant implications, and may function as a benchmark, when using these different corneal tomography devices for the diagnosis, monitoring, and treatment of corneal diseases, especially ectasia and keratoconus. Corneal tomography has also become the gold standard in peri-operative evaluation of laser refractive surgery patients and lens-based refractive surgery patients.

In all the aforementioned clinical practices, the magnitude of change required to be considered true 

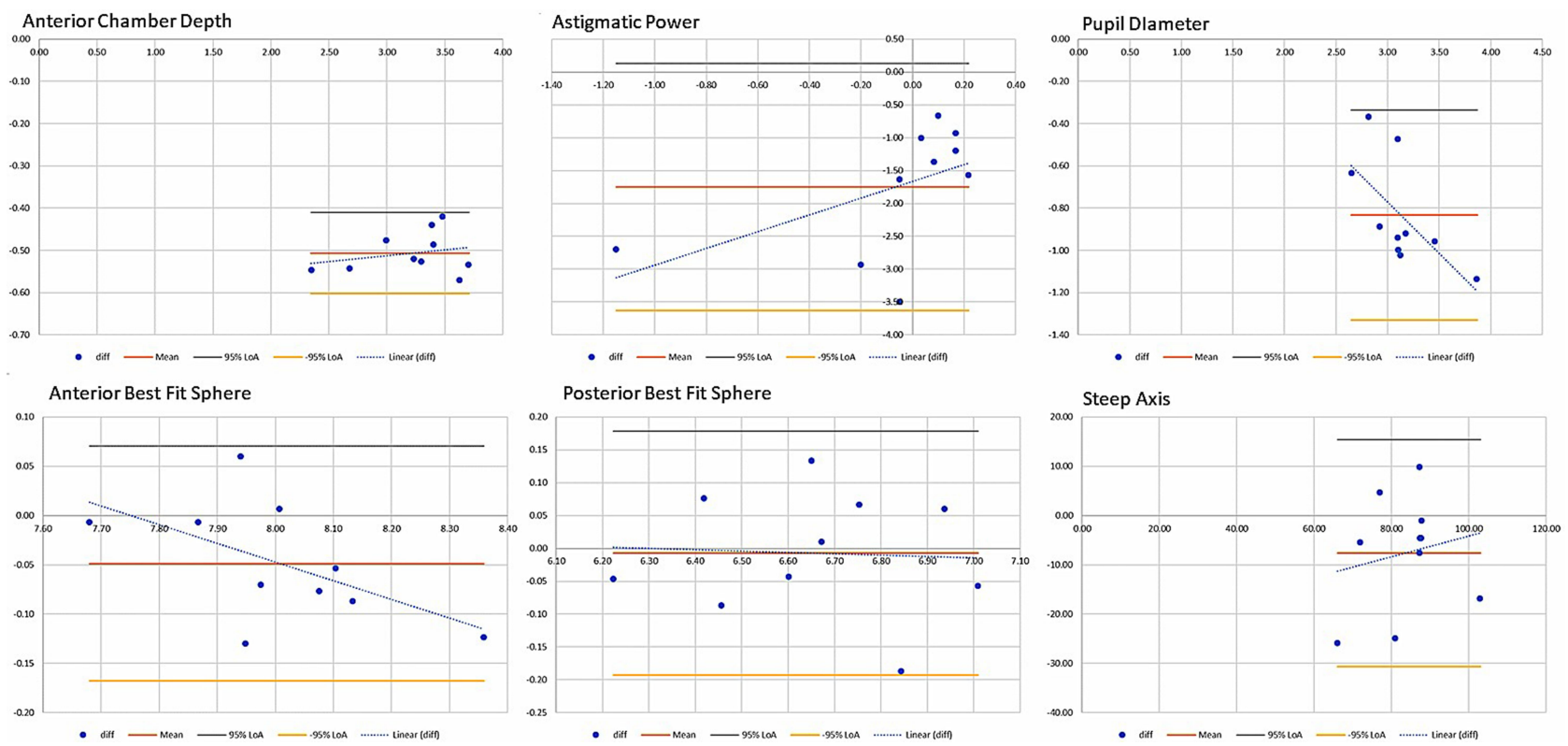

Figure 4 This image illustrates the Bland-Altman plots for the other indices studied, analyzed statistically.

clinical progression rather than either variability due to test re-test variance or poor inter-device agreement should be cautiously established.
Further comparative studies with large samples both of healthy and pathologic corneas should aim to establish a gold standard in corneal tomography which would then

Table 2 Statistical Analysis Correlation, Comparing the Parameters Studied Between the 2 Devices with the Correlation Coefficient $r$, and the 2-Tailed $t$-Test for the Parameters Studied

\begin{tabular}{|l|l|l|}
\hline & $\mathbf{r}$ & $\mathbf{P}$ \\
\hline K2 steep keratometry & 0.94 & 0.022 \\
\hline KI flat keratometry & 0.92 & 0.04 \\
\hline Kmax (steepest) & 0.8 & $>0.05$ \\
\hline X angle kappa & 0.26 & $>0.05$ \\
\hline Y angle kappa & 0.17 & $>0.05$ \\
\hline Kmean power & 0.88 & $>0.05$ \\
\hline BFS front & 0.96 & 0.032 \\
\hline BFS posterior & 0.93 & $>0.05$ \\
\hline ACD & 0.99 & $<0.001$ \\
\hline Astigmatic power & 0.21 & $<0.001$ \\
\hline Steep axis angle & 0.31 & $>0.05$ \\
\hline Pupil diameter & 0.83 & $<0.001$ \\
\hline Thinnest Pachymetry & 0.99 & $<0.001$ \\
\hline Thinnest $x$ from vertex & 0.66 & 0.003 \\
\hline Thinnest $y$ from vertex & 0.23 & $>0.05$ \\
\hline
\end{tabular}


function as a reference both for diagnostic and possibly interventional imaging.

\section{Acknowledgments}

The author wishes to acknowledge Dr: Fillipos Vingopoulos and medical student: Athanasios Zissimopoulos for their statistical expertise offered in this project.

\section{Disclosure}

This work was presented in part as a poster presentation at the 2019 annual AAO meeting, San Francisco, CA, USA.

Dr Kanellopoulos reports being a Consultant for Alcon, Avedro, i-Optics, Keramed, Zeiss, and ISP Surgical; and reports no other potential conflicts of interest for this work.

\section{References}

1. Basmak H, Sahin A, Yildirim N, Papakostas TD, Kanellopoulos AJ. Measurement of angle kappa with synoptophore and Orbscan II in a normal population. J Refract Surg. 2007;23(5):456-460. doi:10.3928/1081-597X-20070501-06

2. Kanellopoulos AJ, Aslanides IM, Asimellis G. Correlation between epithelial thickness in normal corneas, untreated ectatic corneas, and ectatic corneas previously treated with CXL; is overall epithelial thickness a very early ectasia prognostic factor? Clin Ophthalmol. 2012;6:789-800. doi:10.2147/OPTH.S31524

3. Kanellopoulos AJ, Asimellis G. Correlation between central corneal thickness, anterior chamber depth, and corneal keratometry as measured by Oculyzer II and WaveLight OB820 in preoperative cataract surgery patients. J Refract Surg. 2012;28(12):895-900. doi:10.3928/ 1081597X-20121005-07

4. Kanellopoulos AJ, Asimellis G. Anterior segment optical coherence tomography: assisted topographic corneal epithelial thickness distribution imaging of a keratoconus patient. Case Rep Ophthalmol. 2013;4(1):74-78. doi:10.1159/000350630

5. Kanellopoulos AJ, Chiridou M, Asimellis G. Optical coherence tomography-derived corneal thickness asymmetry indices: clinical reference study of normal eyes. J Cataract Refract Surg. 2014;40 (10):1603-1609. doi:10.1016/j.jcrs.2014.01.041

6. Kanellopoulos AJ, Asimellis G, Georgiadou S. Digital pupillometry and centroid shift changes after cataract surgery. J Cataract Refract Surg. 2015;41(2):408-414. doi:10.1016/j.jcrs.2014.05.049

7. Kanellopoulos AJ, Asimellis G. Revisiting keratoconus diagnosis and progression classification based on evaluation of corneal asymmetry indices, derived from Scheimpflug imaging in keratoconic and suspect cases. Clin Ophthalmol. 2013;7:1539-1548. doi:10.2147/OPTH.S44741

8. Kanellopoulos AJ, Asimellis G. Distribution and repeatability of corneal astigmatism measurements (Magnitude and Axis) evaluated with color light emitting diode reflection topography. Cornea. 2015;34(8):937-944. doi:10.1097/ICO.0000000000000476

9. Kanellopoulos AJ, Asimellis G. OCT-derived comparison of corneal thickness distribution and asymmetry differences between normal and keratoconic eyes. Cornea. 2014;33(12):1274-1281. doi:10.1097/ICO.0000000000000275

10. Kanellopoulos AJ, Asimellis G. OCT corneal epithelial topographic asymmetry as a sensitive diagnostic tool for early and advancing keratoconus. Clin Ophthalmol. 2014;18(8):2277-2287. doi:10.2147/ OPTH.S67902
11. Kanellopoulos AJ, Asimellis G. Color light-emitting diode reflection topography: validation of keratometric repeatability in a large sample of wide cylindrical-range corneas. Clin Ophthalmol. 2015;5 (9):245-252. doi:10.2147/OPTH.S68371

12. Kanellopoulos AJ, Asimellis G. Clinical correlation between placido, scheimpflug and LED color reflection topographies in imaging of a scarred cornea. Case Rep Ophthalmol. 2014;5(3):311-317. doi:10.1159/000365962

13. Kanellopoulos AJ, Asimellis G. Clear-cornea cataract surgery: pupil size and shape changes, along with anterior chamber volume and depth changes. A Scheimpflug imaging study. Clin Ophthalmol. 2014;24(8):2141-2150. doi:10.2147/OPTH.S68370

14. Kanellopoulos AJ. Topography-modified refraction (TMR): adjustment of treated cylinder amount and axis to the topography versus standard clinical refraction in myopic topography-guided LASIK. Clin Ophthalmol. 2016;3 (10):2213-2221. doi:10.2147/OPTH.S122345

15. Kanellopoulos AJ, Krueger RR, Asimellis G. Cross-linking and corneal imaging advances. Biomed Res Int. 2015;2015:306439. doi: $10.1155 / 2015 / 306439$

16. Kanellopoulos AJ. Topography-guided custom retreatments in 27 symptomatic eyes. J Refract Surg. 2005;21(5):S513-8. doi:10.3928/ 1081-597X-20050901-19

17. Kanellopoulos AJ, Pe LH, Perry HD, Donnenfeld ED. Modified intracorneal ring segment implantations (INTACS) for the management of moderate to advanced keratoconus: efficacy and complications. Cornea. 2006;25(1):29-33. doi:10.1097/01. ico.0000167883.63266.60

18. Kanellopoulos AJ. Post-LASIK ectasia. Ophthalmology. 2007;114 (6):1230. doi:10.1016/j.ophtha.2007.03.041

19. Kanellopoulos AJ. Comparison of sequential vs same-day simultaneous collagen cross-linking and topography-guided PRK for treatment of keratoconus. $J$ Refract Surg. 2009;25(9):S812-8. doi:10.3928/1081597X-20090813-10

20. Kanellopoulos AJ, Binder PS. Management of corneal ectasia after LASIK with combined, same-day, topography-guided partial transepithelial PRK and collagen cross-linking: the athens protocol. J Refract Surg. 2011;27(5):323-331. doi:10.3928/1081597X-20101105-01

21. Kanellopoulos AJ. Topography-guided hyperopic and hyperopic astigmatism femtosecond laser-assisted LASIK: long-term experience with the $400 \mathrm{~Hz}$ eye-Q excimer platform. Clin Ophthalmol. 2012;6:895-901. doi:10.2147/OPTH.S23573

22. Kanellopoulos AJ, Kahn J. Topography-guided hyperopic LASIK with and without high irradiance collagen cross-linking: initial comparative clinical findings in a contralateral eye study of 34 consecutive patients. $J$ Refract Surg. 2012;28(11 Suppl):S837-40. doi:10.3928/1081597X-20121005-05

23. Kanellopoulos AJ, Asimellis G. Introduction of quantitative and qualitative cornea optical coherence tomography findings induced by collagen cross-linking for keratoconus: a novel effect measurement benchmark. Clin Ophthalmol. 2013;7:329-335. doi:10.2147/ OPTH.S40455

24. Kanellopoulos AJ, Asimellis G. Comparison of Placido disc and Scheimpflug image-derived topography-guided excimer laser surface normalization combined with higher fluence CXL: the Athens Protocol, in progressive keratoconus. Clin Ophthalmol. 2013;7:1385-1396. doi:10.2147/OPTH.S44745

25. Kanellopoulos AJ, Asimellis G. Refractive and keratometric stability in high myopic LASIK with high-frequency femtosecond and excimer lasers. J Refract Surg. 2013;29(12):832-837. doi:10.3928/ 1081597X-20130924-02

26. Kanellopoulos AJ, Asimellis G. Comparison of high-resolution Scheimpflug and high-frequency ultrasound biomicroscopy to anterior-segment OCT corneal thickness measurements. Clin Ophthalmol. 2013;7:2239-2247. doi:10.2147/OPTH.S53718 
27. Kanellopoulos AJ, Asimellis G. Forme fruste keratoconus imaging and validation via novel multi-spot reflection topography. Case Rep Ophthalmol. 2013;4(3):199-209. doi:10.1159/000356123

28. Kanellopoulos AJ, Asimellis G. Keratoconus management: long-term stability of topography-guided normalization combined with high-fluence CXL stabilization (the Athens Protocol). J Refract Surg. 2014;30(2):88-93. doi:10.3928/1081597X-20140120-03

29. Kanellopoulos AJ, Asimellis G. Corneal refractive power and symmetry changes following normalization of ectasias treated with partial topography-guided PTK combined with higher-fluence CXL (the Athens Protocol). J Refract Surg. 2014;30(5):342-346. doi:10.3928/ 1081597X-20140416-03

30. Kanellopoulos AJ, Dupps WJ, Seven I, Asimellis G. Toric topographically customized transepithelial, pulsed, very high-fluence, higher energy and higher riboflavin concentration collagen cross-linking in keratoconus. Case Rep Ophthalmol. 2014;5(2):172-180.

31. Kanellopoulos AJ, Asimellis G. Novel placido-derived topography-guided excimer corneal normalization with cyclorotation adjustment: enhanced athens protocol for keratoconus. $J$ Refract Surg. 2015;31(11):768-773. doi:10.3928/1081597X-20151021-06

32. Kanellopoulos AJ, Asimellis G. LASIK ablation centration: an objective digitized assessment and comparison between two generations of an excimer laser. J Refract Surg. 2015;31(3):164-169. doi:10.3928/ 1081597X-20150225-01

33. Kanellopoulos AJ. Topography-guided LASIK versus small incision lenticule extraction (SMILE) for myopia and myopic astigmatism: a randomized, prospective, contralateral eye study. J Refract Surg. 2017;33(5):306-312. doi:10.3928/1081597X-20170221-01

34. Kanellopoulos AJ. Topography-guided LASIK versus small incision lenticule extraction: long-term refractive and quality of vision outcomes. Ophthalmology. 2018;125(10):1658-1659. doi:10.1016/j. ophtha.2018.04.006

35. Kanellopoulos AJ. Management of progressive keratoconus with partial topography-guided PRK combined with refractive, customized CXL - a novel technique: the enhanced Athens protocol. Clin Ophthalmol. 2019;2(13):581-588. doi:10.2147/OPTH.S188517

36. Kanellopoulos AJ. Ten-year outcomes of progressive keratoconus management with the athens protocol (Topography-guided partial-refraction PRK combined with CXL). J Refract Surg. 2019;35(8):478-483. doi:10.3928/1081597X-20190627-01

37. Kanellopoulos AJ, Vingopoulos F, Sideri AM. Long-term stability with the athens protocol (Topography-guided partial PRK combined with cross-linking) in pediatric patients with keratoconus. Cornea. 2019;38(8):1049-1057. doi:10.1097/ICO.0000000000001996

38. Bourges J-L, Alfonsi N, Laliberté J-F, et al. Average 3-dimensional models for the comparison of orbscan II and pentacam pachymetry maps in normal corneas. Ophthalmology. 2009;116(11):2064-2071.

39. Meyer JJ, Gokul A, Vellara HR, Prime Z, McGhee CNJ. Repeatability and agreement of orbscan II, pentacam HR, and Galilei tomography systems in corneas with keratoconus. Am $J$ Ophthalmol. 2017;175:122-128. doi:10.1016/j.ajo.2016.12.003

40. Kawamorita T, Uozato H, Kamiya K, et al. Repeatability, reproducibility, and agreement characteristics of rotating Scheimpflug photography and scanning-slit corneal topography for corneal power measurement. J Cataract Refract Surg. 2009;35(1):127-133. doi:10.1016/j.jcrs.2008.10.019

41. Rozema JJ, Wouters K, Mathysen DGP, Tassignon M-J. Overview of the repeatability, reproducibility, and agreement of the biometry values provided by various ophthalmic devices. Am J Ophthalmol. 2014;158(6):1111-1120.e1111. doi:10.1016/j.ajo.2014.08.014

Clinical Ophthalmology
42. Randleman JB, Lynn MJ, Perez-Straziota CE, Weissman HM, Kim SW. Comparison of central and peripheral corneal thickness measurements with scanning-slit, scheimpflug and fourier-domain ocular coherence tomography. Br J Ophthalmol. 2015;99(9):1176. doi:10.1136/bjophthalmol-2014-306340

43. Hashemi H, Yekta A, Khabazkhoob M. Effect of keratoconus grades on repeatability of keratometry readings: comparison of 5 devices. J Cataract Refract Surg. 2015;41(5):1065-1072. doi:10.1016/j.jcrs.2014.08.043

44. Lanza M, Paolillo E, Carnevale UA, Lanza A. Central corneal thickness evaluation in healthy eyes with three different optical devices. Cont Lens Anterior Eye. 2015;38(6):409-413. doi:10.1016/j.clae.2015.05.006

45. Lang PZ, Thulasi P, Khandelwal SS, Hafezi F, Randleman JB. Comparing change in anterior curvature after corneal cross-linking using scanning-slit and scheimpflug technology. Am J Ophthalmol. 2018;191:129-134. doi:10.1016/j.ajo.2018.04.018

46. Oliveira C, Ribeiro C, Franco S. Corneal imaging with slit-scanning and Scheimpflug imaging techniques. Clin Exp Optom. 2011;94 (1):33-42. doi:10.1111/j.1444-0938.2010.00509.x

47. Cairns G, Ghee C. Orbscan computerized topography: attributes, applications, and limitations. J Cataract Refract Surg. 2005;31 (1):205-220. doi:10.1016/j.jcrs.2004.09.047

48. Bland J, Altman D. Statistical methods for assessing agreement between two methods of clinical measurement. Lancet. 1986;1:307-310. doi:10.1016/S0140-6736(86)90837-8

49. Bland J, Altman D. Measuring agreement in method comparison studies. Stat Methods Med Res. 1999;8:135-160. doi:10.1177/ 096228029900800204

50. Lackner B, Schmidinger F, Pieh S, Fuvonics M, Skirpik C. Repeatability and repro- ducibility of central corneal thickness measurement with Pentacam, Orbscan, and ultrasound. Optom Vis Sci. 2005;82:892-899. doi:10.1097/01.opx.0000180817.46312.0a

51. Epstein R, Chiu Y, Epstein G. Pentacam HR criteria for curvature change in keratoconus and postoperative LASIK ectasia. J Refract Surg. 2012;28(12):890-894. doi:10.3928/1081597X-20121115-04

52. Guilbert E, Saad A, Grice-Dulac A, Gatinel D. Corneal thickness, curvature and elevation readings in normal corneas: combined Placido-Scheimpflug system versus combined Placido-scanning-slit system. J Cataract Refract Surg. 2012;38(7):1198-1206. doi:10.1016/j.jcrs.2012.01.033

53. Sedaghat M, Daneshvar R, Kargozar A, Derakhshan A, Daraei M. Comparison of central corneal thickness measure- ment using ultrasonic pachymetry, rotating Scheimpflug camera, and scanning-slit topography. Am J Ophthalmol. 2010;150(6):780-789. doi:10.1016/j. ajo.2010.06.013

54. Rosa N, Lanza M, Borelli M, Polito B, Filosa M, Bernardo MD. Comparison of central corneal thickness measured with Orbscan and Pentacam. J Refract Surg. 2007;23(9):895-899. doi:10.3928/1081597X-20071101-05

55. Crawford A, Patel D, Mcghee C. Comparison and repeatability of keratometric and corneal power measurements obtained by Orbscan II, Pentacam, and Galilei corneal tomography systems. Am J Ophthalmol. 2013;156(1):53-60. doi:10.1016/j.ajo.2013.01.029

56. Amano S, Honda H, Amano Y, et al. Comparison of central corneal thickness measurements by rotating Scheimpflug camera, ultrasonic pachymetry, and scanning-slit corneal topography. Ophthalmology. 2006;113(6):937-941. doi:10.1016/j.ophtha.2006.01.063

57. Kim S, Byun Y, Kim E, Kim T. Central corneal thick- ness measurements in unoperated eyes and eyes after PRK for myopia using Pentacam, Orbscan II, and ultrasonic pachymetry. J Refract Surg. 2007;23:888-894. doi:10.3928/1081-597X-20071101-04

Dovepress 


\section{Publish your work in this journal}

Clinical Ophthalmology is an international, peer-reviewed journal covering all subspecialties within ophthalmology. Key topics include: Optometry; Visual science; Pharmacology and drug therapy in eye diseases; Basic Sciences; Primary and Secondary eye care; Patient Safety and Quality of Care Improvements. This journal is indexed on PubMed
Central and CAS, and is the official journal of The Society of Clinical Ophthalmology (SCO). The manuscript management system is completely online and includes a very quick and fair peer-review system, which is all easy to use. Visit http://www.dovepress.com/ testimonials.php to read real quotes from published authors. 